\title{
Well-posedness of the Infinite Prandtl Number Model for Convection with Temperature-Dependent Viscosity
}

\author{
Max Gunzburger Yuki Saka \\ Xiaoming Wang \\ Department of Mathematics and School of Computational Sciences \\ Florida State University, Tallahassee, FL 32306
}

October 6, 2008

\begin{abstract}
We establish the well-posedness of the infinite Prandtl number model for convection with temperature-dependent viscosity, free-slip boundary condition and zero horizontal fluxes.
\end{abstract}

\section{Introduction}

One of the most useful models in fluid and geophysical fluid dynamics is the Boussinesq model for convection which takes the (non-dimensional) form: (see, e.g., [9])

$$
\begin{aligned}
& \frac{1}{P r}\left(\partial_{t} \mathbf{u}+\mathbf{u} \cdot \nabla \mathbf{u}\right)-\nabla \cdot(\nu(\theta) \nabla \mathbf{u})+\nabla p=R a \theta \mathbf{k}, \\
& \nabla \cdot \mathbf{u}=0, \\
& \partial_{t} \theta-\nabla \cdot(k(\theta) \nabla \theta)+\mathbf{u} \cdot \nabla \theta=0,
\end{aligned}
$$

on the domain $\Omega=\mathbb{T}^{2} \times[0,1]$. The temperature $\theta$ satisfies the Dirichlet boundary condition:

$$
\theta(x, y, 0)=1, \quad \theta(x, y, 1)=0
$$

corresponding to heating at the bottom and relative cooling at the top. The velocity $\mathbf{u}$ satisfies the free-slip condition [9]:

$$
\begin{aligned}
& u_{3}(x, y, 1)=u_{3}(x, y, 0)=0 \\
& \partial_{3} u_{1}(x, y, 1)=\partial_{3} u_{1}(x, y, 0)=0 \\
& \partial_{3} u_{2}(x, y, 1)=\partial_{3} u_{2}(x, y, 0)=0
\end{aligned}
$$

which is suitable for many geophysical application where the top and bottom boundaries are somewhat artificial and hence free-slip may be more appropriate to avoid artificial boundary layer [9]. Here $\operatorname{Pr}$ denotes the Prandtl number which is the ratio of the representative value of the kinematic viscosity to the representative value of the thermal diffusivity and $R a$ the Rayleigh number which is the ratio of relative heating to the overall dissipation. Note that, in general, the (nondimensionalized) viscosity $\nu$ and the (non-dimensionalized) thermal diffusivity $k$ depend on the temperature. Such dependence could be of great importance due to the large temperature contrast in certain applications. 
In the mantle flow, the viscosity depends strongly on the temperature. For example, a typical function that is used in the geophysics literature is $\nu(\theta)=\exp (-a|\theta|)[9, \mathrm{p}$. 319], where $a$ quantifies the viscosity contrast, e.g., $\eta=\exp (-a)=\exp (-5 \log (10))=10^{-5}$. We will assume that the viscosity is uniformly bounded and globally Lipschitz, i.e.

$$
\eta \leq \nu(\theta) \leq 1, \quad\left|\nu^{\prime}\right| \leq a
$$

The existence of global weak solution in 3D and strong solution in 2D are established under mild assumptions on the viscosity and diffusivity and no-slip boundary condition [5].

In applications such as mantle convection, the inertial term is relatively small since the Prandtl number is large. Therefore we can formally drop the inertial term in the Boussinesq system and arrive at the following infinite Prandtl number model (for simplicity, we also neglect the temperature dependence of the conductivity)

$$
\begin{aligned}
& -\nabla \cdot(\nu(\theta) \nabla \mathbf{u})+\nabla p=R a \theta \mathbf{k}, \\
& \nabla \cdot \mathbf{u}=0 \\
& \partial_{t} \theta-\Delta \theta+\mathbf{u} \cdot \nabla \theta=0
\end{aligned}
$$

along with the boundary conditions (2) and (3) and with the viscosity $\nu$ depending on $|\theta|$ only. The interested reader may find a rigorous justification of such a model for the case of constant viscosity and no-slip boundary condition [10].

The system as it is postulated is not well-posed since the the solution to the Stokes system is not unique for a given temperature field. Indeed, if $\mathbf{u}$ is a solution to the Stokes problem, $\mathbf{u}+\left(U_{1}, U_{2}, 0\right)$ is also a solution for any constants $U_{1}, U_{2}$ which correspond to averaged horizontal velocities of the fluids, assuming $u_{1}$ and $u_{2}$ have zero averages. These constants cannot be determined from the infinite Prandtl number model directly. However, it is easy to check that these averaged horizontal velocities are constants in time for the full Boussinesq system for convection. Hence we may impose zero horizontal fluxes

$$
\int_{\Omega} u_{1} d \mathbf{x}=\int_{\Omega} u_{2} d \mathbf{x}=0
$$

which is intuitively consistent with the situation in mantle convection.

The temperature-independent viscosity case has been analyzed extensively, e.g., in [2, 3]. A rather detailed analysis is possible in that case since the Stokes equation is linear and hence techniques from potential theory can be used. In fact, in the constant viscosity setting, one of the real challenges is the estimation of a constant called the Nusselt number defined by

$$
N u=\limsup _{T \rightarrow \infty} \frac{1}{T|\Omega|} \int_{0}^{T} \int_{\Omega}|\nabla \theta(\mathbf{x}, t)|^{2} d \mathbf{x} d t
$$

that quantifies the ratio of heat transported due to convection and that due to conduction. Since convection is a nonlinear transport process in our equation, $N u$ also describes the degree of nonlinearity present in our equation and is typically of order of some power of $R a$ (the so-called power law). Estimating the exact value of the power occupies a large part of the current interests of researchers in this area.

The situation is different and more difficult with a temperature dependent viscosity since this introduces a second-order nonlinearity. On top of this, unlike well-studied second-order nonlinearities such as the $p$-Laplacian, what we have here is not monotonic in the temperature variable. Therefore, a variation in the temperature can potentially cause a large variation in the velocity field. The main result of this short note is to show the regularity of the solution to the system we 
consider, and therefore its well-posedness. We will apply classical energy method $[4,8]$ together with an extension of the problem to a periodic domain.

Throughout this note, $\|f\|$ denotes the spatial $L^{2}$ norm of $f, c$ will denote a generic constant depending on the parameters ( $R a$, initial data, the form of viscosity etc) but independent of the solution. We also use $\lesssim$ to denote inequality up to a scalar multiplication.

\section{Well-posedness of the infinite Prandtl number model}

In this section, we show the well-posedness for the system (2)-(6). In order to overcome the difficulty of a second order non-monotonic nonlinearity, we work with a space having higher regularity $\left(H^{2}\right.$ in space). We would usually encounter the difficulty of boundary layers which is circumvented here by an appropriate periodic extension. Such a periodic extension is applicable due to the free-slip boundary condition.

Let $w=\theta-(1-z)$ be a perturbative variable. Then, (5) can be re-expressed in terms of $w$ :

$$
\begin{aligned}
& -\nabla \cdot(\nu(w+(1-z)) \nabla \mathbf{u})+\nabla p=R a \mathbf{k} w \\
& \nabla \cdot \mathbf{u}=0 \\
& \partial_{t} w-\Delta w+\mathbf{u} \cdot \nabla w-u_{3}=0
\end{aligned}
$$

with $w=u_{3}=\partial_{3} u_{1}=\partial_{3} u_{2}=0$ on $z=0,1$, and $\int_{\Omega} u_{1}=\int_{\Omega} u_{2}=0$.

\subsection{Extension to a periodic domain}

In this subsection, we will show that due to the free-slip boundary condition, a solution to (7) can be extended to the periodic domain $\Omega_{e x t}=\mathbb{T}^{2} \times \mathbb{Z}$ and satisfy a slightly modified version of the original equation. Here $\mathbb{Z}=[-1,1]$ with -1 and 1 identified (and hence periodic in $z$ ). On $\Omega$, this new version reduces to the original one. Therefore, our strategy will be to solve a periodic problem on the extended domain with an extended initial data. We then verify that the restriction of the solution of the extended problem to the original domain yields the desired solution of the original problem.

The extension (in the $z$ variable) that we have in mind is odd in $w$ and $u_{3}$ but even in $u_{1}, u_{2}$ and $p$, i.e., we extend $\mathbf{u}, p$, and $w=\theta-(1-z)$ to $\Omega_{e x t}=\mathbb{T}^{2} \times \mathbb{Z}$ in the following way:

$$
\begin{aligned}
& w\left(x_{1}, x_{2},-x_{3}\right)=-w\left(x_{1}, x_{2}, x_{3}\right), \\
& u_{3}\left(x_{1}, x_{2},-x_{3}\right)=-u_{3}\left(x_{1}, x_{2}, x_{3}\right), \\
& u_{2}\left(x_{1}, x_{2},-x_{3}\right)=u_{2}\left(x_{1}, x_{2}, x_{3}\right) \\
& u_{1}\left(x_{1}, x_{2},-x_{3}\right)=u_{1}\left(x_{1}, x_{2}, x_{3}\right) \\
& p\left(x_{1}, x_{2},-x_{3}\right)=p\left(x_{1}, x_{2}, x_{3}\right)
\end{aligned}
$$

We then identify the two ends of $[-1,1]$ to complete the periodic extension and denote the onedimensional torus $\mathbb{Z}$. This means that if $w$ and $u_{3}$ are smooth in $\Omega$, then they are continuous up to the first derivatives across the boundary points $x_{3} \in \mathbb{Z}$ due to their homogeneous Dirichlet boundary conditions. For $u_{1}$ and $u_{2}$, they are continuous across the boundary points $x_{3} \in \mathbb{Z}$ due to the even extension. Since their normal derivative is zero, we also have that they are continuous up to the first derivatives across the boundary points $x_{3} \in \mathbb{Z}$.

Note that the $k$ th order normal derivative of an odd (even) function at a point $x_{0}$ has the same (different) sign as its value at the point $-x_{0}$ if $k$ is odd (even), and the sign flips if $k$ is even (odd). 
Then, it can be easily verified that $\mathbf{u}$ and $w$ satisfy the following system on the extended domain $\Omega_{\text {ext }}=\mathbb{T}^{2} \times \mathbb{Z}$ where $\mathbb{Z}=[-1,1]$ with -1 and 1 identified (and hence periodic in $z$ ).

$$
\begin{aligned}
-\nabla \cdot(\nu(w+g(z)) \nabla \mathbf{u})+\nabla p & =R a \mathbf{k} w \\
\nabla \cdot \mathbf{u} & =0 \\
\partial_{t} w-\Delta w+\mathbf{u} \cdot \nabla w-u_{3} & =0
\end{aligned}
$$

with periodic boundary condition and average zero on $\Omega_{\text {ext }}$. Here $g$ is the odd extension of $1-z$ given by

$$
g(z)=\left\{\begin{array}{cl}
1-z & 0 \leq z \leq 1 \\
-1-z & -1 \leq z<0
\end{array}\right.
$$

so that $g(-z)=-g(z)$.

Although $g$ has a jump discontinuity at $z=0$, the extended $\nu$ does not have any discontinuity since it depends on $|\theta|=|w+g|$ and both $w$ and $g$ are odd and $|w+g|_{z= \pm 0}=1$. An important observation that we will use in the sequel is that the extension of $w$ extends $\nu(w+g)$ in a way that the Hölder continuity and Lipshitz continuity of $\nu$ are preserved.

\section{$2.2 \quad$ Existence}

In this subsection, we obtain several a priori estimates for the solutions of (8) with a goal of showing existence.

The following lemma is straightforward from the Stokes equation and the assumption that the viscosity is bounded below (independent of $\theta$ ).

Lemma 2.1 If $\boldsymbol{u}$ solves (8), then

$$
\|\nabla \boldsymbol{u}\| \leq C\|w\|, \forall t
$$

Proof We test the Stokes system with $\mathbf{u}$ to obtain

$$
\begin{aligned}
\eta \int_{\Omega_{e x t}}|\nabla \mathbf{u}|^{2} & \leq \int_{\Omega_{e x t}} \nu(\theta)|\nabla \mathbf{u}|^{2} \\
& \leq R a \int_{\Omega_{e x t}} w u_{3} \\
& \leq R a\|w\|\left\|u_{3}\right\| \\
& \leq c R a\|w\|\left\|\partial_{3} u_{3}\right\| .
\end{aligned}
$$

The Poincaré inequality is applicable due to the zero average assumption.

It is also easy to derive the following standard a priori estimate for solutions to the extended system (8).

\section{Lemma 2.2}

$$
\|w\|_{L^{\infty}\left(0, T ; L^{2}\right)}+\|w\|_{L^{2}\left(0, T ; H^{1}\right)} \leq c .
$$

Proof We multiply the temperature equation by $w$ and integrate over $\Omega_{e x t}$ to deduce

$$
\begin{aligned}
\frac{1}{2} \int_{\Omega_{e x t}} \partial_{t}\left(w^{2}\right)+\|\nabla w\|^{2} & =\int_{\Omega_{e x t}} u_{3} w \\
& \leq\left\|u_{3}\right\|\|w\| \\
& \leq c\left\|\nabla u_{3}\right\|\|w\| \\
& \leq c\|w\|^{2}
\end{aligned}
$$


where we have used Lemma 2.1 and the Poincaré inequality which is valid for $u_{3}$ since we enforce the average zero condition.

The desired result follows from the Poincaré inequality (applicable due to the average zero assumption on $w$ ) and the Gronwall inequality.

These estimates apply to $\theta$ as well since $\theta$ and $w$ differ from each other by $1-z$.

Next we obtain an estimate for the time derivative of $w$.

\section{Lemma 2.3}

$$
\left\|\partial_{t} w\right\|_{L^{2}\left(0, T ; H^{-1}\right)} \lesssim 1+\|\nabla w\|_{L^{2}\left(0, T ; L^{2}\right)} .
$$

Proof For $\phi \in H_{p e r}^{1}\left(\Omega_{e x t}\right)$ with average zero, we have

$$
\begin{aligned}
\int \phi \partial_{t} w & =-\int \nabla w \cdot \nabla \phi+\int\left(w \mathbf{u} \cdot \nabla \phi+u_{3} \phi\right) \\
& \lesssim\left(\|\nabla w\|+\left\|u_{3}\right\|+\left(\int(\mathbf{u} w)^{2}\right)^{1 / 2}\right)\|\nabla \phi\| \\
& \lesssim\left(\|\nabla w\|+\left\|u_{3}\right\|+\|\mathbf{u}\|_{L^{6}}\|w\|_{L^{3}}\right)\|\nabla \phi\| \\
& \lesssim\left(\|\nabla w\|+\left\|u_{3}\right\|+\|\nabla \mathbf{u}\|\|w\|^{\frac{1}{2}}\|\nabla w\|^{\frac{1}{2}}\right)\|\nabla \phi\| \\
& \lesssim(1+\|\nabla w\|)\|\nabla \phi\|
\end{aligned}
$$

where we have used the previous lemmas $\left(L^{\infty}\left(H^{1}\right)\right.$ on $\mathbf{u}$ and Poincaré inequality on $\left.w\right)$. The desired result follows from this inequality.

These lemmas lead to the existence of a weak solution for $w(0) \in L^{2}$ which can be rigorously verified using standard Galerkin method together with the Aubin-Lions compactness lemma [4, 8] which implies the strong convergence of the approximate $w$ in $L^{q}\left(L^{2}\right), q \in[0, \infty)$. Thus we have the following theorem on the existence of weak solutions. Details can be found in [6].

Theorem 2.4 For $w(0) \in L^{2}\left(\Omega_{\text {ext }}\right), \int_{\Omega_{e x t}} w(0)=0$, there exists $(\boldsymbol{u}, w)$ such that

1. $w \in L^{\infty}\left(0, T ; L^{2}\right) \cap L^{2}\left(0, T ; H_{p e r}^{1}\right), \int_{\Omega_{e x t}} w=0$,

2. $\partial_{t} w \in L^{2}\left(0, T ; H^{-1}\right),\left.w\right|_{t=0}=w(0)$,

3. $\boldsymbol{u} \in L^{\infty}\left(0, T ; H_{p e r}^{1}\right), \int_{\Omega_{e x t}} \boldsymbol{u}=0, \nabla \cdot \boldsymbol{u}=0$

and satisfies the weak formulation:

$$
\begin{aligned}
\int_{\Omega_{e x t}} \nu(w+g(z)) \nabla \boldsymbol{u}: \nabla \boldsymbol{v} & =R a \int_{\Omega_{e x t}} w v_{3} \\
\int_{\Omega_{e x t}} \partial_{t} w \psi+\int_{\Omega_{e x t}} \nabla w \cdot \nabla \psi+\int_{\Omega_{e x t}} \boldsymbol{u} \cdot \nabla w \psi-\int_{\Omega_{e x t}} u_{3} \psi & =0
\end{aligned}
$$

for almost all $t$ and all $(\boldsymbol{v}, \psi) \in\left(H_{p e r}^{1}\right)^{3} \times H_{\text {per }}^{1}$ such that $\nabla \cdot \boldsymbol{v}=0$ and $\int_{\Omega_{e x t}} \boldsymbol{v}=0, \quad \int_{\Omega_{e x t}} \psi=0$. 


\section{$3 \quad$ Stability and Uniqueness}

In the case of a temperature independent viscosity, the well-posedness can be shown easily from what has so far been proved. This is not necessarily so for the temperature dependent case because fluctuations of the velocity now depends strongly on fluctuations of the temperature. We can have a glimpse into the strong dependence on the temperature field through the following stability result.

Lemma 3.1 For any $\left(w_{i}, \boldsymbol{u}_{i}, p_{i}\right), i=1,2$ solutions to (8), the following inequality holds:

$$
\left\|\left(w_{1}-w_{2}\right)(t)\right\|^{2} \leq\left\|\left(w_{1}-w_{2}\right)(0)\right\|^{2} \exp \left(c \int_{0}^{t}\left(1+\left\|\nabla w_{2}(\tau)\right\|+\left\|\nabla w_{2}(\tau)\right\|^{2}\left\|\nabla^{2} \boldsymbol{u}_{2}(\tau)\right\|^{4}\right) d \tau\right) .
$$

Proof Let $\left(w_{i}, \mathbf{u}_{i}, p_{i}\right), i=1,2$ be solutions to (8). Let $\zeta=w_{1}-w_{2}$ and $\mathbf{v}=\mathbf{u}_{1}-\mathbf{u}_{2}$. Then,

$$
\partial_{t} \zeta+\mathbf{u}_{1} \cdot \nabla \zeta+\mathbf{v} \cdot \nabla w_{2}-v_{3}-\Delta \zeta=0 .
$$

Testing against $\zeta$ we obtain

$$
\int \frac{1}{2} \partial_{t}|\zeta|^{2}+\int|\nabla \zeta|^{2}=\int \mathbf{v} w_{2} \cdot \nabla \zeta+\int v_{3} \zeta
$$

so that

$$
\begin{aligned}
\int \partial_{t}|\zeta|^{2}+\int|\nabla \zeta|^{2} & \lesssim \int\left|\mathbf{v} w_{2}\right|^{2}+\left\|v_{3}\right\|^{2} \\
& \lesssim\|\mathbf{v}\|_{L^{6}}^{2}\left\|w_{2}\right\|_{L^{3}}^{2}+\left\|v_{3}\right\|^{2} \\
& \lesssim\|\nabla \mathbf{v}\|^{2}\left\|w_{2}\right\|\left\|\nabla w_{2}\right\|+\left\|v_{3}\right\|^{2} \\
& \lesssim\|\nabla \mathbf{v}\|^{2}\left(1+\left\|\nabla w_{2}\right\|\right)
\end{aligned}
$$

where we have applied the Poincaré inequality and the $L^{\infty}\left(L^{2}\right)$ estimates on the perturbative temperature.

From the velocity equation we have

$$
-\nabla \cdot\left(\nu\left(w_{1}+g\right) \nabla \mathbf{v}\right)-\nabla \cdot\left(\left(\nu\left(w_{2}+g\right)-\nu\left(w_{1}+g\right)\right) \nabla \mathbf{u}_{2}\right)+\nabla\left(p_{1}-p_{2}\right)=R a \mathbf{k} \zeta .
$$

Note that due to the Gagliardo-Nirenberg and Sobolev inequalities,

$$
\int|\zeta|^{2}|\nabla \mathbf{u}|^{2} \leq\|\zeta\|_{3}^{2}\|\nabla \mathbf{u}\|_{6}^{2} \leq C\|\zeta\|\|\nabla \zeta\|\left\|\nabla^{2} \mathbf{u}\right\|^{2} .
$$

Therefore, testing the difference of the velocity equations against $\mathbf{v}$, and utilizing the global Lipschitz assumption on the viscosity, we have

$$
\begin{aligned}
\eta \int|\nabla \mathbf{v}|^{2} & \leq \int \nu\left(w_{1}+g\right)|\nabla \mathbf{v}|^{2} \\
& =R a \int \zeta v_{3}-\int\left(\nu\left(w_{2}+g\right)-\nu\left(w_{1}+g\right)\right) \nabla \mathbf{u}_{2} \cdot \nabla \mathbf{v} \\
& \leq R a \int \zeta v_{3}+\frac{a^{2}}{\eta} \int \zeta^{2}\left|\nabla \mathbf{u}_{2}\right|^{2}+\frac{\eta}{4} \int|\nabla \mathbf{v}|^{2} \\
& \leq \eta^{-1} R a^{2}\|\zeta\|^{2}+\frac{\eta}{4} \int\left|\nabla v_{3}\right|^{2}+C \eta^{-1} a^{2}\|\zeta\|\|\nabla \zeta\|\left\|\nabla^{2} \mathbf{u}_{2}\right\|^{2}+\frac{1}{4} \int \nu\left(w_{1}+g\right)|\nabla \mathbf{v}|^{2} \\
& \leq \eta^{-1} R a^{2}\|\zeta\|^{2}+C \eta^{-1} a^{2}\|\zeta\|\|\nabla \zeta\|\left\|\nabla^{2} \mathbf{u}_{2}\right\|^{2}+\frac{1}{2} \int \nu\left(w_{1}+g\right)|\nabla \mathbf{v}|^{2} .
\end{aligned}
$$


Hence,

$$
\frac{d}{d t}\|\zeta\|^{2}+\|\nabla \zeta\|^{2} \lesssim\left(1+\left\|\nabla w_{2}\right\|+\left\|\nabla w_{2}\right\|^{2}\left\|\nabla^{2} \mathbf{u}_{2}\right\|^{4}\right)\|\zeta\|^{2} .
$$

The result then follows by Gronwall's inequality.

Note the terms in the exponential factor. The presence of the $\left\|\nabla^{2} \mathbf{u}_{2}\right\|$ term is specific to the temperature dependent viscosity case; therefore, our aim in the next section is to show that this term is bounded which further implies the uniqueness of course.

\subsection{Regularity}

In this subsection we show that a weak solution has further regularity provided that the initial data belongs to $H_{p e r}^{2}\left(\Omega_{e x t}\right)$. We can approach this in many different ways. Ideally, in each estimation process, we would obtain a bound that is as tight as possible because such estimates might be used to improve the Nusselt number bound. This goal is not met in this paper and remains a subject for future research.

We will first consider the global regularity of the temperature field.

Lemma 3.2 Let $w=\theta-(1-z)$ be a solution to the extended infinite Prandtl number model on the extended domain $\Omega_{\text {ext }}$. Then

$$
\left\|\nabla^{2} w(t)\right\|^{2}+\int_{0}^{t} e^{-C(\tau-t)}\left\|\nabla^{3} w(\tau)\right\|^{2} d \tau \lesssim e^{C t}\left(\left\|\nabla^{2} w(0)\right\|^{2}+1\right) .
$$

Proof In the following, we present the necessary a priori estimates which can be justified rigorously through Galerkin approximation if needed [6].

We take the Laplacian of the temperature equation in (8) and test against $\psi$ to obtain

$$
\int_{\Omega_{e x t}}\left(\psi \partial_{t} \Delta w+(\nabla \Delta w) \cdot \nabla \psi+(\mathbf{u} \cdot \nabla \Delta w) \psi+2\left(\nabla \mathbf{u}: \nabla^{2} w\right) \psi+(\Delta \mathbf{u} \cdot \nabla w) \psi-\psi \Delta u_{3}\right) d \mathbf{x}=0 .
$$

Setting $\psi=\Delta w$, we obtain, after utilizing the periodicity in all directions,

$$
\frac{1}{2} \frac{d}{d t}\|\Delta w\|^{2}+\|\nabla \Delta w\|^{2}+\int\left(2\left(\nabla \mathbf{u}: \nabla^{2} w\right) \Delta w+(\Delta \mathbf{u} \cdot \nabla w) \Delta w-\Delta u_{3} \Delta w\right)=0
$$

Integrating by parts in the fourth and the fifth term and applying the Cauchy-Schwarz inequality we deduce

$$
\begin{aligned}
\frac{1}{2} \frac{d}{d t}\|\Delta w\|^{2}+\|\nabla \Delta w\|^{2} & \lesssim \int_{\Omega_{e x t}}\left(\left|\nabla \mathbf{u}\left\|\left.\nabla^{2} w\right|^{2}+|\nabla \mathbf{u}||\nabla w||\nabla \Delta w|+\left|\nabla \mathbf{u} \| \nabla^{2} w\right|^{2}+\left|\nabla u_{3}\right||\nabla \Delta w|\right) d \mathbf{x}\right.\right. \\
& \lesssim \int_{\Omega_{e x t}}\left(|\nabla \mathbf{u}|^{2}|\nabla w|^{2}+\left|\nabla \mathbf{u} \| \nabla^{2} w\right|^{2}+\left|\nabla u_{3}\right|^{2}\right) d \mathbf{x}+\frac{1}{2}\|\nabla \Delta w\|^{2} \\
& \lesssim\|\nabla w\|_{\infty}^{2}\|\nabla \mathbf{u}\|^{2}+\|\nabla \mathbf{u}\|\left\|\nabla^{2} w\right\|_{4}^{2}+\|\nabla \mathbf{u}\|^{2}+\frac{1}{2}\|\nabla \Delta w\|^{2} \\
& \lesssim\left\|\nabla^{2} w\right\|\left\|\nabla^{3} w\right\|\|\nabla \mathbf{u}\|^{2}+\|\nabla \mathbf{u}\|\left\|\nabla^{2} w\right\|^{\frac{1}{2}}\left\|\nabla^{3} w\right\|^{\frac{3}{2}}+\|\nabla \mathbf{u}\|^{2}+\frac{1}{2}\|\nabla \Delta w\|^{2}
\end{aligned}
$$

where we have also applied the Agmon and Gagliardo-Nirenberg type inequalities

$$
\|\nabla w\|_{\infty} \lesssim\left\|\nabla^{2} w\right\|^{\frac{1}{2}}\left\|\nabla^{3} w\right\|^{\frac{1}{2}}, \quad \text { and } \quad\left\|\nabla^{2} w\right\|_{4} \lesssim\left\|\nabla^{2} w\right\|^{\frac{1}{4}}\left\|\nabla^{3} w\right\|^{\frac{3}{4}} .
$$


Therefore, when combined with the $L^{\infty}\left(H^{1}\right)$ estimates on the velocity and elliptic regularity, we deduce

$$
\frac{d}{d t}\|\Delta w\|^{2}+\|\nabla \Delta w\|^{2} \lesssim\left\|\nabla^{2} w\right\|^{2}+1
$$

which implies the desired result by Gronwall's inequality.

This a priori estimate implies the $L^{\infty}\left(H^{2}\right)$ a priori estimate on the velocity field which would further imply uniqueness.

Lemma 3.3 For solution to the infinite Prandtl number model, we have

$$
\|\boldsymbol{u}\|_{L^{\infty}\left(0, T ; H^{2}\right)} \lesssim\left\|\nabla^{2} w\right\|_{L^{\infty}\left(0, T ; H^{2}\right)}^{2}+1 .
$$

Proof Note that since the extended $\nu$ has a matching derivative at the origin, we can take the weak derivative of the extended viscosity $\nu(\theta)$. Therefore, we have the following weak formulation

$$
\int \nu\left(w+g\left(x_{3}\right)\right) \nabla \partial_{k} \mathbf{u}: \nabla \mathbf{v}+\partial_{k} \nu\left(w+g\left(x_{3}\right)\right) \nabla \mathbf{u}: \nabla \mathbf{v}=R a \int \partial_{k} w v_{3}
$$

Setting $\mathbf{v}=\partial_{k} \mathbf{u}$, we obtain

$$
\begin{aligned}
\eta \int\left|\nabla \partial_{k} \mathbf{u}\right|^{2} & \leq \int\left|\partial_{k} \nu(w+g)\right||\nabla \mathbf{u}|\left|\nabla \partial_{k} \mathbf{u}\right|+R a \int|w|\left|\partial_{k k} u_{3}\right| \\
& \leq \frac{1}{\eta} \int\left|\partial_{k} \nu(w+g)\right|^{2}|\nabla \mathbf{u}|^{2}+\frac{\eta}{4} \int\left|\nabla \partial_{k} \mathbf{u}\right|^{2}+\eta^{-1} R a^{2} \int|w|^{2}+\frac{\eta}{4} \int\left|\partial_{k k} u_{3}\right|^{2} .
\end{aligned}
$$

Exactly as in the proof of Lemma 3.1, we have

$$
\begin{aligned}
\int\left|\partial_{k} \nu(w+g)\right|^{2}|\nabla \mathbf{u}|^{2} & \leq|\nu|_{\text {Lip }}^{2} \int\left|\partial_{k}\right| w+g \|^{2}|\nabla \mathbf{u}|^{2} \\
& \lesssim a\left(\|\nabla w\|_{6}^{2}+1\right)\|\nabla \mathbf{u}\|_{3}^{2} \\
& \lesssim a\left(\left\|\nabla^{2} w\right\|^{2}+1\right)\|\nabla \mathbf{u}\|\left\|\nabla^{2} \mathbf{u}\right\| .
\end{aligned}
$$

Consequently,

$$
\eta\left\|\nabla \partial_{k} \mathbf{u}\right\|^{2} \leq c\left(\left\|\nabla^{2} w\right\|^{2}+1\right)^{2}+\frac{\eta}{2}\left\|\nabla^{2} \mathbf{u}\right\|^{2} .
$$

Combining the estimates for all directions $k$ gives the result.

Combining the a priori estimates we deduce the well-posedness of the periodic problem (with zero mean). The well-posedness of the original problem also follows and we have the following main result.

Theorem 3.4 Suppose that the extended initial data belongs to $H_{p e r}^{2}\left(\Omega_{\text {ext }}\right)$, then there exists a unique solution to the infinite Prandtl number system (8) in the extended domain. As a consequence, if the the initial data for the original problem (7) belongs to $H^{2}(\Omega)$, then there exists a unique solution to the infinite Prandtl number model under the zero horizontal fluxes constraint (6). 
Proof We have already proved the first half of the theorem regarding the periodic case on the extended domain $\Omega_{\text {ext }}$.

Now suppose $\left\|\nabla^{2} w(0)\right\|_{L^{2}(\Omega)}<\infty$ and satisfies the homogeneous Dirichlet boundary conditions, we extend $w$ to $\Omega$ oddly in $z=x_{3}$. It is easy to see that the extended $w(0)$ belongs to $H_{p e r}^{2}\left(\Omega_{e x t}\right)$ by going through the Fourier representation in $x$ and $y$ if necessary. Therefore the result on the periodic case applies. In particular we will have a unique solution $w \in$ $L^{\infty}\left(0, T ; H_{\text {per }}^{2}\left(\Omega_{\text {ext }}\right)\right) \bigcap L^{2}\left(0, T ; H_{\text {per }}^{3}\left(\Omega_{\text {ext }}\right)\right)$ and $\mathbf{u} \in L^{\infty}\left(0, T ;\left(H_{\text {per }}^{2}\left(\Omega_{\text {ext }}\right)\right)^{3}\right)$.

Due to the special structure of the system, it is easy to see that $-w(t ; x, y,-z), p(t ; x, y,-z)$, $\left(u_{1}(t ; x, y,-z), u_{2}(t ; x, y,-z),-u_{3}(t ; x, y,-z)\right)$ is also a solution to the extended system with the same initial data. Therefore, $w$ and $u_{3}$ are odd in $z=x_{3}$ while $u_{1}$ and $u_{2}$ are even in $z=x_{3}$ by the uniqueness. This implies that

$$
\begin{aligned}
\left.w\right|_{z=0} & =0 \\
\left.u_{3}\right|_{z=0} & =0, \\
\left.\partial_{3} u_{j}\right|_{z=0} & =0, \quad j=1,2 .
\end{aligned}
$$

The boundary condition at $z=1$ can be verified similarly.

Hence the restriction of $w$ and $\mathbf{u}$ onto $\Omega$ solves the original initial boundary value problem (7).

\section{Conclusion and Remarks}

In this paper, we proved the well-posedness of the infinite Prandtl number model for convection with a temperature dependent viscosity under zero horizontal flux assumption. With an application to mantle convection in mind, we considered a free-slip boundary condition and reformulated the equation in a periodic domain.

The infinite Prandtl number model with free-slip boundary condition without fixing the horizontal fluxes is not well-posed due to non-uniqueness. Indeed, the procedure that we presented above can be repeated to show the existence of solution for the same (non-linear) Stokes equations coupled with the following temperature equation

$$
\partial_{t} w-\Delta w+\mathbf{u} \cdot \nabla w-u_{3}+U_{1} \partial_{x} w+U_{2} \partial_{y} w=0
$$

where $U_{1}, U_{2}$ represent averaged horizontal velocities while $\mathbf{u}$ has zero mean in the horizontal directions.

The existence of a weak solution can be shown without the periodic extension (and hence can be applied to other boundary conditions as well). In this case, in the weak formulation $(9,10)$ we should use $\Omega$ instead of $\Omega_{e x t}$, and the space that we should use for velocity is $V=\{\mathbf{v} \in$ $\left(H^{1}(\Omega)\right)^{3} \mid \mathbf{v}$ periodic in $\left.x, y ; \nabla \cdot \mathbf{v}=0 ; \int_{\Omega} v_{1} d \mathbf{x}=\int_{\Omega} v_{2} d \mathbf{x}=0 ;\left.v_{3}\right|_{z=0,1}=0\right\}$ and for the perturbative temperature $w$ we use $H_{p e r, 0}^{1}=\left\{w \in H^{1}(\Omega) \mid w\right.$ periodic in $\left.x, y ;\left.w\right|_{z=0,1}=0\right\}$. More details can be found in $[6]$.

The assumption that the viscosity depends on the absolute value of the temperature is not essential either. In fact, we can show a weak maximum principle for the temperature field for the weak solution. Hence if the initial data is uniformly bounded in space (bounded below by zero in particular for physical relevance), we can show the existence of a weak solution that is uniformly bounded (below by zero in particular) [6].

The well-posedness of the problem is the first step towards further investigation of convection in temperature dependent viscosity environment. For instance, we need to consider enhancement 
of heat transport in the vertical direction due to convection over conduction (quantified by the Nusselt number $[2,3]$ ). An upper bound on the Nusselt number for the model under investiagtion which scales like $R a^{\frac{1}{2}}$ was derived in [6]. We also need to consider accurate and effective numerical schemes for the system, especially long time behaviors (see [1] for the case of constant viscosity and long time behavior, [7] for finite time approximation).

We only considered the case of free-slip which is relevant for mantle convection. The free-slip boundary condition allows us to study the problem in a periodic setting after a periodic extension. Other physically interesting boundary conditions such as no-slip and mixed free-slip/no-slip still need to be investigated. Also, we have focused on the temperature dependence of the viscosity while neglecting the temperature dependence of the conductivity which fits common belief in the geophysical community [9]. It would be interesting to consider temperature dependent viscosity and conductivity and study the sensitivity of the solutions with respect to the temperature in order to determine the relative importance of the dependence on the temperature.

\section{Acknowledgements}

This work is supported in part by grants from the National Science Foundation under DMS0549368

and DMS0606671. This manuscript is based on a part of the Ph.D thesis of Yuki Saka under the direction of the other two authors.

\section{References}

[1] W. Cheng and X. Wang, A Uniformly Dissipative Scheme for Stationary Statistical Properties of The Infinite Prandtl Number Model, Applied Mathematics Letters, 21 (2008) 1281-1285. DOI: 10.1016/j.aml.2007.07.036

[2] P. Constantin and C.R. Doering, On upper bounds for infinite Prandtl number convection with or without rotation Journal of Mathematical Physics, Volume 42, Issue 2, February 2001, pp.784-795

[3] C.R. Doering, F. Otto, and M.G. Reznikoff, Bounds on vertical heat transport for infinite Prandtl number Rayleigh-Bénard convection, J. Fluid Mech. (2006), vol. 560, pp.229-241.

[4] J.L. Lions, Quelques méthodes de re'solution des problémes aux limites non linéaires Dunod 1968.

[5] S. A. Lorca, and J. L. Boldrini, The initial value problem for a generalized Boussinesq model Nonlinear Analysis 36, 1999 457-480.

[6] Y. Saka, Analysis of Two Partial Differential Equation Models in Fluid Mechanics: Nonlinear Spectral Eddy-Viscosity Model of Turbulence and Infinite-Prandtl-Number Model of Mantle Convection, Ph.D Thesis, Florida State University, 2007.

[7] M. Tabata and D. Tagami, Error estimates of finite element methods for nonstationary thermal convection problems with temperature-dependent coefficients, Numerische Mathematik, Vol. 100, No.2, pp. 351-372, 2005.

[8] R. Temam, Navier-Stokes Equations, Theory and Numerical Analysis, Studies in Mathematics and Ints Applications, No. 2. North-Holland, Amsterdam-New York-Oxford 1977. 
[9] D L. Turcotte, and G. Schubert, Geodynamics Applications of Continuum Physics to Geological problems John Wiley and Sons, 1982.

[10] X. Wang, Infinite Prandtl number limit of Rayleigh-Benard convection CPAM, 57, (2004) no.10, p.1265-1282. 